\title{
Fertility preservation in post-pubescent female cancer patients: A practical guideline for clinicians
}

\author{
AMIRRTHA SRIKANTHAN ${ }^{1}$, EITAN AMIR ${ }^{1}$, PHILIPPE BEDARD $^{1}$, \\ MEREDITH GIULIANI ${ }^{2}$, DAVID HODGSON ${ }^{2}$, STEPHANIE LAFRAMBOISE $^{3}$, ANCA PRICA ${ }^{1}$, \\ KAREN YEE $^{1}$, ELLEN GREENBLATT ${ }^{4}$, JEREMY LEWIN ${ }^{1}$ and ABHA GUPTA ${ }^{1,5}$ \\ Departments of ${ }^{1}$ Medical Oncology and Hematology, ${ }^{2}$ Radiation Oncology, ${ }^{3}$ Gynecologic Oncology, \\ Princess Margaret Cancer Centre, Toronto, ON M5G 2M9; ${ }^{4}$ Mount Sinai Centre for Fertility \\ and Reproductive Health, Mount Sinai Hospital, Toronto, ON M5T 2Z5; ${ }^{5}$ Division \\ of Haematology/Oncology, The Hospital for Sick Children, Toronto, ON M5G 1X8, Canada
}

Received June 7, 2017; Accepted October 30, 2017

DOI: $10.3892 / \mathrm{mco} .2017 .1486$

\begin{abstract}
Increasing accessibility of fertility preservation (FP) options has permitted women to retain fertility following anticancer therapies. Several published guidelines have made recommendations for FP however their implementation into practice is currently unknown. In this review, we aim to provide oncology clinicians practical information about FP options for post-pubescent female cancer patients and recommendations for care delivery in order to answer preliminary questions and help triage whether FP referral is appropriate. Herein, we present a resource for oncology providers to guide them with FP discussions. Key points that are discussed in this critical review include: i) All cancer patients beginning a new plan of care should be informed of potential fertility risk. ii) If a woman requests further information on FP interventions, referral to a FP clinic should be made. iii) Given the evolving technologies in this area, patients should be informed of those which are proven and unproven, with oocyte and embryo preservation recognized as standard practice. iv) Random start (independent of menstrual cycle day) techniques are available to minimize oncologic treatment delays. v) Specific protocols for ovarian stimulation may be center-specific. vi) There is unlikely an increased cancer recurrence risk as a result of stimulation protocols in women with hormone-sensitive cancers. vii) Lastly, given the absence of consensus in the literature, routine use of GnRH analogs is not recommended for all cancer patients, however may be considered in select cases, such as high-risk women in whom definitive FP is not possible or feasible.
\end{abstract}

Correspondence to: Dr Abha Gupta, Division of Haematology/Oncology, The Hospital for Sick Children, 555 University Avenue, Toronto, ON M5G 1X8, Canada

E-mail: abha.gupta@sickkids.ca

Key words: fertility preservation, females, cancer, guidelines

\section{Introduction}

Modern cancer therapies have led to increasing survival for young women with cancer (1). Many of these treatments can lead to detrimental impact on a woman's fertility, such as alkylating agents and radiation therapy $(1,2)$. As more young women with cancer survive, issues such as fertility preservation increase in importance. This is evident from the number of publications and guidelines that have been recently published (2-6). Despite these recommendations, the provision of fertility preservation remains low $(7,8)$. Lack of health care provider knowledge has been documented as a possible reason for lack of fertility preservation discussions and referrals.

The purpose of this guideline is to provide oncology healthcare providers with practical information about fertility preservation (FP) for post-pubescent female cancer patients. This work was undertaken at the Princess Margaret Cancer Center (PM) in Toronto, Canada, a large adult academic cancer centre, to provide a practical resource to health care providers in busy oncology clinics. This document is organized based on common topics that arise during acute treatment oncology care. The target readership for this guideline are physicians, nurse practitioners, nurses, pharmacists and social workers involved in cancer care delivery.

\section{Data collection methods}

A systematic literature review of published literature from January 2000 to June 2014 was completed using MEDLINE with search items: fertility preservation and cancer, cancer and female fertility, and fertility preservation and reviews. The most recent international oncology guidelines regarding FP in female cancer patients were also identified for which these practical guidelines were drafted upon. All recommendations were drawn from the literature search, with the available evidence provided to reviewing content experts. Once completed, eight subspecialty content experts at PM critiqued and reviewed the guidelines, to ensure agreement. After the initial review, revisions were made and recirculated to the content experts for final review and endorsement. 
Recommendations in this guideline are graded as per the GRADE system (Grading of Recommendations, Assessment, Development and Evaluation) (9). In GRADE, grades have two components: a two-level representation of the strength of recommendation (strong or weak) and a four-level representation of the quality of the evidence (high, moderate, low, and very low).

\section{Results}

Infertility defined. The two major categories of infertility relating to cancer treatment include acute ovarian failure (AOF) and premature (or primary) ovarian insufficiency or failure (POI/POF) (10). Patients who lose ovarian function during cancer therapy or shortly after its completion are classified as having acute ovarian failure (AOF). Patients, who retain ovarian function after therapy completion and then experience the trio of amenorrhea, hypoestrogenism and hypergonadotropism before the age of 40, are classified as having POI (10). POI and AOF are not mutually exclusive and it is possible for a patient to have both diagnoses. POI and AOF exist along the same disease gradient, and it is possible that those with AOF have residual ovarian activity, or may regain ovarian activity. An individual's true ovarian reserve prior to anti-cancer treatment and post treatment cannot be determined with complete accuracy (1).

Quantifying the risk of infertility. Estimating the risk of infertility in women after cancer therapy depends on several patient factors including: cancer profile (type, site of disease), general health, baseline fertility and patient age at exposure to gonadotoxic agents and patient's current age (11). Treatment factors that influence fertility include: total cumulative dose of alkylating agents, cumulative gonadal toxicity of multi-drug chemotherapy regimens, radiotherapy location and dose, and surgery to reproductive organs $(3,12)$. The risk can be summarized as the chance of $\mathrm{AOF}$ with treatment, or $\mathrm{POI} / \mathrm{POF}$ in the years following treatment. The known and potential fertility risks of all cancer treatments should be considered when counseling patients regarding fertility impact. The decision to pursue FP intervention is the patient's choice and will be influenced by the estimated risk to fertility and current patient age (3). Although the success for FP in women over 40 is low (13), information is key, and discussions regarding risk to fertility should occur irrespective of age. The likelihood of achieving successful preservation if pursued is a secondary issue. Patients with the potential for POF should be advised that the biggest challenge remains the uncertainty regarding estimating the optimal time window for oocyte preservation and balancing costs of preservation of fertilized embryos versus unfertilized oocytes. For example, for an unpartnered woman who currently has adequate ovarian function, she must choose between preserving oocytes now, or waiting until she is ready to preserve embryos (either with donor and/or partner sperm).

Timing of fertility discussion. There are at least two points in the cancer journey that fertility should be discussed: i) at diagnosis and ii) at the end of primary cancer treatment. All newly diagnosed cancer patients and patients beginning a new plan of care should be informed of the potential risk (if any) of the proposed treatment plan to their fertility as a standard part of a treatment and consent discussion (recommendation: Strong, level of evidence: Low) (3). Patients may require immediate initiation of cancer therapy or may be too ill to undergo FP procedures, but, regardless, should be informed of the potential risks to fertility and be made aware of the reason(s) why she is not a suitable candidate for FP interventions. Documentation of this fertility discussion into the patient chart, prior to onset of treatment, should take place (recommendation: Strong, level of evidence: Low) (3).

A reproductive specialist or health care provider should be involved to discuss FP interventions if a patient requests this information (recommendation: Strong, level of evidence: Low). Patients should be informed of all FP options, including proven and unproven technologies being evaluated through trials (recommendation: Strong, level of evidence: Low) (3). These discussions can happen with various members of the health care teams including nurse navigators (when available), primary oncologists, or reproductive specialists. The decision to pursue FP interventions is up to the patient and family; declining to pursue any intervention is always acceptable.

As there may be an opportunity to preserve fertility in women following the completion of cancer therapy, women who may still be interested in childbearing should be sent for formal ovarian function assessment through their local fertility clinic approximately 6 months after completion of anti-cancer therapy.

FP interventions available for women. Available FP technologies and interventions for females are listed in Table I.

Usefulness of gonadotropin-releasing hormone (GnRH) analogs in preserving fertility. Gonadal protection through hormonal manipulation with GnRH analogs for FP in women treated with gonadotoxic chemotherapy has demonstrated conflicting results $(3,14-26)$. Published clinical guidelines from clinical associations are variable in their recommendations regarding GnRH analogs. The American Society of Clinical Oncology does not support the use of GnRH analogs; however, the Canadian Fertility and Andrology Society supports their use in FP $(3,27)$. Patients and families should be made aware of this treatment and the conflicting data in the literature regarding the use of $\mathrm{GnRH}$ analogs to protect ovarian function. Emerging evidence in the breast cancer population suggest potential benefit with increased pregnancy rates post treatment (28), however, the study had limitations, including the use of surrogate endpoints with poor correlation to ovarian function, and lack of information on assisted reproductive technology usage. Given the absence of consensus in the literature, routine use of GnRH analogs is not recommended for the purpose of fertility preservation, however may be considered in select cases, especially in high-risk women in whom definitive FP is not possible or feasible (recommendation: Strong, level of evidence: Moderate).

Potential benefit of ovarian transposition (oophoropexy). Oophoropexy is a treatment strategy to move ovaries away from the radiation field when pelvic radiation is performed $(3,12,29)$. Due to radiation scatter and post-procedure migration, ovaries 
Table I. Fertility preservation strategies dependent on type of treatment.

If the treatment includes

Cancer surgery

Radiation therapy to pelvis and gonads

Cytotoxic treatment with high gonadotoxicity

Endocrine therapy for estrogen sensitive breast cancer
Consider the following options

1. Fertility sparing surgery (gonad preservation)

2. Uterus preservation

3. Cryopreservation if high risk of gonadal damage anticipated

1. Shielding to reduce damage to ovaries

2. Ovarian transposition

3. Cryopreservation if high risk of gonadal damage anticipated

1. Cryopreservation

1. Cryopreservation if age at completion of treatment is at a time when natural fertility is likely low

- >33 years of age at start of treatment if 5 years planned

- >28 years of age at start of treatment if 10 years planned

Adapted from Rodriguez-Wallberg and Oktay, 2014 (12).

are not always protected and patients should be advised of the possibility of lack of success (3). The procedure should be performed as close to the time of radiation treatment onset due to risk of re-migration of the ovaries (30), and can be done laparoscopically or through a laparotomy $(31,32)$. Concerns have been raised regarding the efficacy of this procedure, as success, measured by preservation of short-term menstrual function, is $\sim 50 \%$ (33). The main reasons for failure include radiation scatter, risk to the blood supply of the ovary during this procedure, which in itself may diminish ovarian function over time, and re-migration $(31,32)$.

Spontaneous pregnancies have been reported in women with transposed ovaries, without the need for re-positioning of ovaries (34). However, if infertility develops and in vitro fertilization is needed after ovarian transposition, oocyte retrieval may be more complicated (35). Either a second procedure is needed to reposition the ovaries to the pelvis (34), or egg collection is performed percutaneously with the risk of reducing the efficiency of this procedure (35). Other risks include ovarian dysfunction leading to ovarian cysts and the theoretical risk of increased difficulty diagnosing ovarian cancer if the ovaries are no longer palpable on bimanual examination (33). Thus, transposition may be helpful to offer some ovarian protection, especially if oocyte harvesting is not practical prior to the initiation of therapy, albeit with the aforementioned caveats.

Feasibility of ovarian tissue cryopreservation (OTC) and re-transplantation. OTC is a process in which strips of ovarian cortical tissue are harvested by minimally invasive laparoscopic surgery under a general anesthetic. OTC and future transplantation is a potential option for women who are unable to undertake ovarian stimulation and traditional transvaginal oocyte retrieval. This technique may be considered, particularly in women who are anyways undergoing pelvic surgery, who are then destined to require adjuvant pelvic radiation therapy. The amount of tissue required for optimal FP (i.e., the whole ovary or merely strips from the ovarian cortex) is unclear; however, typically the cortex of an entire ovary is preserved in adult patients (33). The ovarian tissue may be utilized in two ways: i) tissue may be thawed and re-implanted at a later date in order to reconstitute ovarian reserve, or ii) alternatively oocytes from cortical strips could be harvested to be used in IVF.

OTC proves advantageous as it does not require ovarian stimulation, with less delay to starting cancer therapy; however, this technique is considered experimental (33), remains an unproven technology, and requires operating room and anesthesia time. There are only a few centers worldwide demonstrating successful live births using re-implanted OTC (36). Referred patients must be medically fit and able to undergo a laparoscopic abdominal procedure.

The biggest issue with OTC remains the growing and substantial concern regarding the reintroduction of malignant or pre-malignant cells with re-implanted ovarian tissue. This is of exceptional consideration in patients with hematologic malignancies (37), and is contraindicated in patients with leukemia (38), with extreme caution still required in solid tumors (39-41). Ideally, consideration for this procedure should be undertaken only in the context of a clinical trial, where follow-up for and reporting of recurrent cancer and pregnancies is feasible (33).

Embryo and/or oocyte cryopreservation (OC). Ideally, OC and/or embryo preservation should be offered prior to cancer therapy. If this is not feasible, it then should be considered following the completion of cancer therapy, however ovarian reserves may be depleted and stimulation/harvest may not be successful (3). If post-therapy preservation is being considered, a 6-month washout is recommended following chemotherapy (recommendation: Strong, level of evidence: Low). OC should occur before the onset of cytotoxic therapy in patients at high risk for treatment-related AOF (recommendation: Strong, level of evidence: Moderate) (3), such as in women greater than age 35 at diagnosis. OC and embryo preservation are both considered established, non-experimental FP methods (recommendation: Strong, level of evidence: Low). Embryo preservation requires sperm (either from a partner or sperm bank) which is used to fertilize the oocyte prior to freezing $(3,12)$. 
Table II. Ovarian stimulation protocol timelines.

Timeline

Procedure

Baseline

Day 3-12

Day 5 post stimulation

Day 8-12 post stimulation

$36 \mathrm{~h}$ after $\mathrm{HCG}$ injection
Tests are conducted prior to any treatment. A baseline ultrasound examination and a blood test (usually for FSH, estradiol and AMH). Cycle day is determined by the clinic.

Ovarian stimulation. Daily injectable fertility medications begin on a day that is chosen by the cycle coordinator.

Cycle monitoring. Regular office visits start and are continued every 1-2 days until follicle aspiration.

Ovulation induction. Ovulation is triggered with an injection of HCG, or a GnRH agonist, administered when the follicles are judged to be mature based on ultrasound and hormone criteria. Egg retrieval. Women undergo an aspiration procedure that takes $<30 \mathrm{~min}$.

Women arrive at the clinic $1 \mathrm{~h}$ before the scheduled retrieval procedure and go home approximately $2 \mathrm{~h}$ afterwards.

AMH, anti-mullerian hormone; FSH, follicle-stimulating hormone; GnRH, gonadotropin-releasing hormone; HCG, human chorionic gonadotropin. Adapted from Rodriguez-Wallberg and Oktay, 2014 (12).

Both embryo and OC require referral to a fertility/reproductive health clinic. Procedures are often undertaken under light sedation through a transvaginal approach. Patients need to be medically fit with stable blood counts. Transmittable disease screening (syphilis screen, hepatitis $C$ virus serology, hepatitis B surface antigen, HIV1/HIV2 serology) is required prior to undergoing any procedures.

Length of $O C$. Previously, oocyte preservation could take up to 6 weeks to complete depending on the phase of menstrual cycle. This option may not be suitable for patients with clinical conditions that preclude a delay in starting therapy (recommendation: Strong, level of evidence: Low) $(3,12,42,43)$. Controlled ovarian stimulation requires daily subcutaneous injections for 9-13 days and multiple transvaginal ultrasounds and blood tests, and transvaginal aspiration under mild sedation. Now, 'random start' protocols are becoming increasingly available which preclude cycle dependency and shorten the preservation time to 10-14 days; thus, women may not need to be at the beginning of a menstrual cycle at the time of stimulation (44). Random stimulation is currently a newer technique and may not be available at all fertility clinics. Stimulation protocol decisions will be made by the fertility clinic with input from the treating oncologist regarding urgency of treatment. It is important to note that most women will not have enough time to pursue more than one cycle of harvesting, and so, should the first cycle fail or lead to poor oocyte retrieval, additional cycles may not be possible. Patients should be seen as soon as possible by a fertility specialist to minimize delays.

Typical ovarian simulation protocol. Previously, concern has been raised regarding the safety of ovarian stimulation in hormone-sensitive malignancies and cancer recurrence. Limited by selection bias and short-term follow-up, available data do not indicate increased cancer recurrence risk as a result of stimulation protocols (recommendation: Strong, level of evidence: Low) (45).

Oocyte stimulation protocols generally involve the use of 3-4 types of drugs: i) A medication (a GnRH analogue) to suppress the luteinizing hormone (LH) surge and ovulation (until the developing eggs are ready); ii) a follicle stimulating hormone (FSH) product to stimulate development of multiple eggs; iii) an LH containing product may be added to the regimen; iv) human chorionic gonadotropin or a $\mathrm{GnRH}$ agonist trigger to cause final maturation of the eggs (46). Typical timelines a patient may experience are identified in Table II.

Likelihood of successful pregnancy after oocyte or embryo freezing. Pregnancy rates with embryo and $\mathrm{OC}$ are specific to individual fertility clinics (47). Subsequent successful pregnancies are dependent on the quality of the eggs, which is inversely related to female age at retrieval (44). Success is also related to the thawing process, the ability to fertilize the thawed oocyte (for those who underwent OC alone) and the ability of the implanted embryos to develop into pregnancies (48). Overall pregnancy rates for OC range from 7-25\% (48).

Embryo freezing has a long history and many IVF centers are able to maintain live-birth rates of $>25 \%$ after transfer of embryos created from eggs collected from women under 36 years of age. Success rates fall significantly as patients move beyond this age threshold (44). The overall IVF clinical pregnancy rate in Canada from embryo preservation is $32 \%$ per cycle started, $34 \%$ per egg retrieval procedure, and $39 \%$ per embryo transfer procedure. This does not take into account the thawing process (47).

Tamoxifen. In addition to agents with potential gonadotoxicity, consideration should be given to the impact of agents which delays a woman's ability to consider pregnancy due to prolonged exposure to agents with teratogenic potential. For example, pre-menopausal women with breast cancer may require adjuvant endocrine therapy, such as with tamoxifen, which, while is not directly gonadotoxic, is teratogenic and women taking this medication should not attempt pregnancy. As tamoxifen is used for a duration of 5-10 years in the adjuvant setting, a woman's fertility is affected by the negative impact of increasing age while receiving tamoxifen (12). 
Specifically, the woman's anticipated age at the end of treatment is an important factor to consider during decisions to pursue FP interventions at diagnosis. For example, if a woman's age upon tamoxifen completion is 30, she may wish to postpone oocyte cryopreservation until then. However, if age upon completion is greater than 35 , she may rather choose to harvest prior to initiation of tamoxifen (49). There is limited evidence to guide clinicians regarding the safety of temporary cessation of tamoxifen in order to attempt pregnancy. An international clinical trial is currently underway to evaluate this question, the results of which will be very important for women facing this challenging situation (POSITIVE: A study evaluating the pregnancy outcomes and safety of interrupting endocrine therapy for young women with endocrine responsive breast cancer who desire pregnancy) (50). For the moment, individual oncologists should encourage and create an environment receptive to open dialogue with their patients, to balance the potential risks of holding tamoxifen for conception and gestation with the desire of pregnancy and focus on the substantial uncertainties regarding risk estimates.

Impacts on discussions regarding FP. Multiple strategies exist to address fertility discussions and facilitation of FP referrals including the provision of written materials (51), web-based decision aids $(52,53)$, the development of dedicated clinical programs (54), and the identification of a fertility preservation network with access to infertility expertise at respective oncology centers (54). Whenever possible, team members who have the time and expertise to have the conversations should be identified in order to maximize opportunities for consistent information delivery to patients.

In conclusion, delivery of FP to female cancer patients who desire such treatments can be challenging. This guideline provides practical information, recommendations and strategies on the implementation of FP for practicing oncology teams to use in real-time when seeing their patients. Discussion of infertility risk is recommended for all patients who may be affected as part of the standard consent process at the time of treatment discussion. Whenever possible, conduits to permit ongoing discussion to help with decision-making should be implemented in cancer programs to ensure equal access to information.

\section{References}

1. Donnez J and Dolmans MM: Fertility preservation in women. Nat Rev Endocrinol 9: 735-749, 2013.

2. Roberts J, Ronn R, Tallon $\mathrm{N}$ and Holzer H: Fertility preservation in reproductive-age women facing gonadotoxic treatments. Curr Oncol 22: e294-e304, 2015.

3. Loren AW, Mangu PB, Beck LN, Brennan L, Magdalinski AJ, Partridge AH, Quinn G, Wallace WH and Oktay K; American Society of Clinical Oncology: Fertility preservation for patients with cancer: American Society of Clinical Oncology clinical practice guideline update. J Clin Oncol 31: 2500-2510, 2013.

4. Muñoz M, Santaballa A, Seguí MA, Beato C, de la Cruz S, Espinosa J, Fonseca PJ, Perez J, Quintanar T and Blasco A: SEOM Clinical Guideline of fertility preservation and reproduction in cancer patients (2016). Clin Transl Oncol 18: 1229-1236, 2016.

5. Font-Gonzalez A, Mulder RL, Loeffen EA, Byrne J, van Dulmen-den Broeder E, van den Heuvel-Eibrink MM, Hudson MM, Kenney LB, Levine JM, Tissing WJ, et al; PanCareLIFE Consortium: Fertility preservation in children adolescents, and young adults with cancer: Quality of clinical practice guidelines and variations in recommendations. Cancer 122: 2216-2223, 2016.
6. Goldfarb SB, Kamer SA, Oppong BA, Eaton A, Patil S, Junqueira MJ, Olcese C, Kelvin JF and Gemignani ML: Fertility Preservation for the Young Breast Cancer Patient. Ann Surg Oncol 23: 1530-1536, 2016

7. Srikanthan A, Amir E and Warner E: Does a dedicated program for young breast cancer patients affect the likelihood of fertility preservation discussion and referral? Breast 27: 22-26, 2016.

8. Lewin J, Ma JMZ, Mitchell L, Tam S, Puri N, Stephens D, Srikanthan A, Bedard P, Razak A, Crump M, et al: The positive effect of a dedicated adolescent and young adult fertility program on the rates of documentation of therapy-associated infertility risk and fertility preservation options. Support Care Cancer 25: 1915-1922, 2017

9. Atkins D, Best D, Briss PA, Eccles M, Falck-Ytter Y, Flottorp S, Guyatt GH, Harbour RT, Haugh MC, Henry D, et al; GRADE Working Group: Grading quality of evidence and strength of recommendations. BMJ 328: 1490, 2004.

10. Metzger ML, Meacham LR, Patterson B, Casillas JS, Constine LS, Hijiya N, Kenney LB, Leonard M, Lockart BA, Likes W, et al: Female reproductive health after childhood, adolescent, and young adult cancers: Guidelines for the assessment and management of female reproductive complications. J Clin Oncol 31: 1239-1247, 2013.

11. Iwase A, Nakamura T, Nakahara T, Goto M and Kikkawa F: Anti-Müllerian hormone and assessment of ovarian reserve after ovarian toxic treatment: A systematic narrative review. Reprod Sci 22: 519-526, 2015.

12. Rodriguez-Wallberg KA and Oktay K: Fertility preservation during cancer treatment: Clinical guidelines. Cancer Manag Res 6: 105-117, 2014

13. Ouhilal S, Lachgar H, Bissonnette F, Buckett B, Kadoch I, St-Michel P and Mahutte N: Public funding of IVF without age limits: A cautionary tale from Quebec. Fertil Steril 104: e207, 2015.

14. Chen H, Li J, Cui T and Hu L: Adjuvant gonadotropin-releasing hormone analogues for the prevention of chemotherapy induced premature ovarian failure in premenopausal women. Cochrane Database Syst Rev (11): CD008018, 2011.

15. Clowse ME, Behera MA, Anders CK, Copland S, Coffman CJ, Leppert PC and Bastian LA: Ovarian preservation by GnRH agonists during chemotherapy: A meta-analysis. J Womens Health (Larchmt) 18: 311-319, 2009.

16. Kim SS, Lee JR, Jee BC, Suh CS, Kim SH, Ting A and Petroff B: Use of hormonal protection for chemotherapy-induced gonadotoxicity. Clin Obstet Gynecol 53: 740-752, 2010.

17. Del Mastro L, Ceppi M, Poggio F, Bighin C, Peccatori F, Demeestere I, Levaggi A, Giraudi S, Lambertini M, D'Alonzo A, et al: Gonadotropin-releasing hormone analogues for the prevention of chemotherapy-induced premature ovarian failure in cancer women: Systematic review and meta-analysis of randomized trials. Cancer Treat Rev 40: 675-683, 2014

18. Badawy A, Elnashar A, El-Ashry $M$ and Shahat M: Gonadotropin-releasing hormone agonists for prevention of chemotherapy-induced ovarian damage: Prospective randomized study. Fertil Steril 91: 694-697, 2009.

19. Bedaiwy MA, Abou-Setta AM, Desai N, Hurd W, Starks D, El-Nashar SA, Al-Inany HG and Falcone T: Gonadotropin-releasing hormone analog cotreatment for preservation of ovarian function during gonadotoxic chemotherapy: a systematic review and metaanalysis. Fertil Steril 95: 906-914 e1-4, 2011.

20. Yang B, Shi W, Yang J, Liu H, Zhao H, Li X and Jiao S: Concurrent treatment with gonadotropin-releasing hormone agonists for chemotherapy-induced ovarian damage in premenopausal women with breast cancer: A meta-analysis of randomized controlled trials. Breast 22: 150-157, 2013.

21. Sverrisdottir A, Nystedt M, Johansson $\mathrm{H}$ and Fornander T: Adjuvant goserelin and ovarian preservation in chemotherapy treated patients with early breast cancer: Results from a randomized trial. Breast Cancer Res Treat 117: 561-567, 2009.

22. Gerber B, von Minckwitz G, Stehle H, Reimer T, Felberbaum R, Maass N, Fischer D, Sommer HL, Conrad B, Ortmann O, et al; German Breast Group Investigators: Effect of luteinizing hormone-releasing hormone agonist on ovarian function after modern adjuvant breast cancer chemotherapy: The GBG 37 ZORO study. J Clin Oncol 29: 2334-2341, 2011.

23. Del Mastro L, Boni L, Michelotti A, Gamucci T, Olmeo N, Gori S, Giordano M, Garrone O, Pronzato P, Bighin C, et al: Effect of the gonadotropin-releasing hormone analogue triptorelin on the occurrence of chemotherapy-induced early menopause in premenopausal women with breast cancer: A randomized trial. JAMA 306: 269-276, 2011. 
24. Munster PN, Moore AP, Ismail-Khan R, Cox CE, Lacevic M, Gross-King M, Xu P, Carter WB and Minton SE: Randomized trial using gonadotropin-releasing hormone agonist triptorelin for the preservation of ovarian function during (neo)adjuvant chemotherapy for breast cancer. J Clin Oncol 30: 533-538, 2012.

25. Elgindy EA, El-Haieg DO, Khorshid OM, Ismail EI, Abdelgawad M, Sallam HN and Abou-Setta AM: Gonadatrophin suppression to prevent chemotherapy-induced ovarian damage: A randomized controlled trial. Obstet Gynecol 121: 78-86, 2013.

26. Demeestere I, Brice P, Peccatori FA, Kentos A, Dupuis J, Zachee P, Casasnovas O, Van Den Neste E, Dechene J, De Maertelaer V, et al: No evidence for the benefit of gonadotropinreleasing hormone agonist in preserving ovarian function and fertility in lymphoma survivors treated with chemotherapy: Final long-term report of a prospective randomized trial. J Clin Oncol 34: 2568-2574, 2016.

27. Wallace WH, Thomson AB, Saran F and Kelsey TW: Predicting age of ovarian failure after radiation to a field that includes the ovaries. Int J Radiat Oncol Biol Phys 62: 738-744, 2005

28. Moore HC, Unger JM, Phillips KA, Boyle F, Hitre E, Porter D, Francis PA, Goldstein LJ, Gomez HL, Vallejos CS, et al; POEMS/S0230 Investigators: Goserelin for ovarian protection during breast-cancer adjuvant chemotherapy. N Engl J Med 372: 923-932, 2015

29. Terenziani M, Piva L, Meazza C, Gandola L, Cefalo G and Merola M: Oophoropexy: a relevant role in preservation of ovarian function after pelvic irradiation. Fertil Steril 91: 935 e15-6, 2009.

30. Williams RS, Littell RD and Mendenhall NP: Laparoscopic oophoropexy and ovarian function in the treatment of Hodgkin disease. Cancer 86: 2138-2142, 1999.

31. Barahmeh S, Al Masri M,Badran O, Masarweh M,El-Ghanem M, Jaradat I and Lataifeh I: Ovarian transposition before pelvic irradiation: Indications and functional outcome. J Obstet Gynaecol Res 39: 1533-1537, 2013.

32. Moawad NS, Santamaria E, Rhoton-Vlasak A and Lightsey JL: Laparoscopic Ovarian Transposition Before Pelvic Cancer Treatment: Ovarian Function and Fertility Preservation. J Minim Invasive Gynecol 24: 28-35, 2017.

33. Lee SJ, Schover LR, Partridge AH, Patrizio P, Wallace WH, Hagerty K, Beck LN, Brennan LV and Oktay K; American Society of Clinical Oncology: American Society of Clinical Oncology recommendations on fertility preservation in cancer patients. J Clin Oncol 24: 2917-2931, 2006

34. Morice P, Thiam-Ba R, Castaigne D, Haie-Meder C, Gerbaulet A, Pautier P, Duvillard P and Michel G: Fertility results after ovarian transposition for pelvic malignancies treated by external irradiation or brachytherapy. Hum Reprod 13: 660-663, 1998.

35. Zinger M, Liu JH, Husseinzadeh N and Thomas MA: Successful surrogate pregnancy after ovarian transposition, pelvic irradiation and hysterectomy. J Reprod Med 49: 573-574, 2004.

36. Practice Committee of American Society for Reproductive Medicine: Ovarian tissue cryopreservation: A committee opinion. Fertil Steril 101: 1237-1243, 2014.

37. Dolmans MM, Jadoul P, Gilliaux S, Amorim CA, Luyckx V, Squifflet J, Donnez J and Van Langendonckt A: A review of 15 years of ovarian tissue bank activities. J Assist Reprod Genet 30 : 305-314, 2013.

38. Bastings L, Beerendonk CC, Westphal JR, Massuger LF, Kaal SE, van Leeuwen FE, Braat DD and Peek R: Autotransplantation of cryopreserved ovarian tissue in cancer survivors and the risk of reintroducing malignancy: A systematic review. Hum Reprod Update 19: 483-506, 2013.

39. Luyckx V, Durant JF, Camboni A, Gilliaux S, Amorim CA, Van Langendonckt A, Irenge LM, Gala JL, Donnez J and Dolmans MM: Is transplantation of cryopreserved ovarian tissue from patients with advanced-stage breast cancer safe? A pilot study. J Assist Reprod Genet 30: 1289-1299, 2013.

40. Sørensen SD, Greve T, Wielenga VT, Wallace WH and Andersen CY: Safety considerations for transplanting cryopreserved ovarian tissue to restore fertility in female patients who have recovered from Ewing's sarcoma. Future Oncol 10: 277-283, 2014

41. Rodríguez-Iglesias B, Novella-Maestre E, Herraiz S, Díaz-García C, Pellicer N, Pellicer A: New methods to improve the safety assessment of cryopreserved ovarian tissue for fertility preservation in breast cancer patients. Fertil Steril 104 1493-1502. e2, 2015
42. Fernbach A,Lockart B, Armus CL, Bashore LM,Levine J,Kroon L, Sylvain G and Rodgers C: Evidence-Based Recommendations for Fertility Preservation Options for Inclusion in Treatment Protocols for Pediatric and Adolescent Patients Diagnosed With Cancer. J Pediatr Oncol Nurs 31: 211-222, 2014.

43. Ginsberg JP: Educational paper: The effect of cancer therapy on fertility, the assessment of fertility and fertility preservation options for pediatric patients. Eur J Pediatr 170: 703-708, 2011.

44. Koch $\mathrm{J}$ and Ledger W: Ovarian stimulation protocols for oncofertility patients. J Assist Reprod Genet 30: 203-206, 2013.

45. Azim AA, Costantini-Ferrando M and Oktay K: Safety of fertility preservation by ovarian stimulation with letrozole and gonadotropins in patients with breast cancer: A prospective controlled study. J Clin Oncol 26: 2630-2635, 2008.

46. Cakmak H and Rosen MP: Random-start ovarian stimulation in patients with cancer. Curr Opin Obstet Gynecol 27: 215-221, 2015.

47. Canadian Fertility and Andrology Society: Human Assisted Reproduction 2013 Live Birth Rates for Canada. http://www.cfas. ca/index.php?option $=$ com_content $\&$ view $=$ article $\& i d=1205 \% 3$ Alive-birth-rates-2012\&catid $=929 \% 3$ Apress-releases\&Itemid $=130$. Accessed August 21, 2014.

48. Cil AP, Bang H and Oktay K: Age-specific probability of live birth with oocyte cryopreservation: an individual patient data meta-analysis. Fertil Steril 100: 492-499 e3, 2013.

49. Valentini A, Finch A, Lubinski J, Byrski T, Ghadirian P, Kim-Sing C, Lynch HT, Ainsworth PJ, Neuhausen SL, Greenblatt E, et al: Chemotherapy-induced amenorrhea in patients with breast cancer with a BRCA1 or BRCA2 mutation. J Clin Oncol 31: 3914-3919, 2013.

50. NCIC Clinical Trials Group: NCIC Clinical Trials. Breast Disease Site. 2015; Available from: https://www.ctg.queensu.ca/ public/breast/breast-disease-site.

51. Hill KA, Nadler T, Mandel R, Burlein-Hall S, Librach C, Glass K and Warner E: Experience of young women diagnosed with breast cancer who undergo fertility preservation consultation. Clin Breast Cancer 12: 127-132, 2012.

52. Garvelink MM, Ter Kuile MM, Louwé LA, Hilders CG and Stiggelbout AM: A Delphi consensus study among patients and clinicians in the Netherlands on the procedure of informing young breast cancer patients about Fertility Preservation. Acta Oncol 51: 1062-1069, 2012.

53. Peate M, Meiser B, Cheah BC, Saunders C, Butow P, Thewes B, Hart R, Phillips KA, Hickey M and Friedlander M: Making hard choices easier: A prospective, multicentre study to assess the efficacy of a fertility-related decision aid in young women with early-stage breast cancer. Br J Cancer 106: 1053-1061, 2012.

54. Loren AW, Brazauskas R, Chow EJ, Gilleece M, Halter J, Jacobsohn DA, Joshi S, Pidala J, Quinn GP, Wang Z, et al: Physician perceptions and practice patterns regarding fertility preservation in hematopoietic cell transplant recipients. Bone Marrow Transplant 48: 1091-1097, 2013. 